\title{
Modeling of photochemical processes in continuous-flow reactors
}

\author{
Anca Roibu, Simon Kuhn* \\ Department of Chemical Engineering, KU Leuven, Celestijnenlaan 200F, 3001 Leuven, \\ Belgium \\ *E-mail: simon.kuhn@kuleuven.be
}

\subsection{Introduction}

Despite the progress in the past decades in the area of light-driven reactions, only a limited number of photochemical reactions is implemented at large scale. ${ }^{[1,2]}$ The major hurdle which needs to be overcome is linked to the scale-up strategy. ${ }^{[3]}$ The limited light penetration (described by Lambert-Beer's law) rapidly decreases the transformation efficiency in large-scale batch reactors as only non-uniform light intensities are achieved in the vessel. However, using continuous flow reactors provides an avenue for efficient scale-up. Moreover, the characteristic reactor sizes on the micro- or milli-scale allow for a homogeneous light distribution in the reacting volume and advanced designs ensure sufficient productivity at the desired scale. ${ }^{[4]}$

Another reason is the difficulty of modeling photochemical reactors. ${ }^{[3]}$ Scaling-up can be realized by starting working with a laboratory-scale reactor and continue by gradually increasing the reactor size until reaching the desired productivity or by predicting the performance of the large scale reactor with mathematical modeling. Modeling is more precise and cost efficient, but is characterized by a higher complexity. ${ }^{[5,6]}$ Consequently, most pilot-scale photoreactors are still designed using empirical or semiempirical methods. ${ }^{[7]}$ Predicting the performance of a large-scale reactor of a different geometry than the one investigated in the laboratory requires knowing the intrinsic kinetic parameters of the reaction. ${ }^{[6,8]}$ Alfano and Cassano described a methodology for scaling-up photoreactors which uses modeling at small and large scale: firstly for determination of the kinetic parameters and then for predicting the pilot-scale reactor performance. ${ }^{[8]}$

This chapter aims to introduce the main concepts of a holistic scale-up strategy based on combined modeling and experiments. We will highlight the important steps of photoreactor modeling such as the kinetic model, radiance, mass and momentum balance etc., and which are the variables to be exchanged between them to ensure efficient and robust scaling. Furthermore, examples from various application fields of photochemistry, e.g. water treatment and synthetic chemistry, will be offered to illustrate the implementation of the described concepts. This chapter will appeal to both chemical engineers and chemists aiming to develop modeling as a tool for scaling-up photochemical reactors.

\subsection{Modeling continuous photochemical processes for photoreactor scaling-up}

Alfano and Cassano reported a scale-up methodology based on photoreactor modeling by coupling the radiation, mass and momentum balances with the kinetic model. ${ }^{[8]}$ Figure 1.1 presents the main steps of this method and was adapted from the flow scheme reported by Ghafoori. ${ }^{[6]}$ 
The scale-up approach encompasses two parts: one involves using a laboratory-scale reactor and the second a large-scale reactor. The laboratory scale reactor is used to determine the intrinsic kinetic parameters; their use allows the possibility to implement the reaction in a photoreactor of a different size, geometry and operated under different conditions. ${ }^{[6]}$ The first step is to determine the mechanism of the investigated reaction, as the kinetic model is designed based on the reaction scheme. Alfano and Cassano recommend to have a validated kinetic model and highlight the importance of fundamental chemistry research to obtain reliable results. ${ }^{[8]}$ In the next steps, the amount of absorbed photons determined from the radiance balance is included in the kinetic model, which is integrated further in the material balance. The intrinsic parameters are estimated after optimizing the algorithm, so the errors between the predictions of the model and the experimental results are minimized.

Once the kinetic parameters are determined, they are included in the same kinetic model which is coupled with the radiation balance characteristic of the large-scale reactor. The reaction rate determined in this way is integrated in the mass balance together with the momentum balance. The momentum balance is not present in the case of the laboratory-scale reactor because it is recommended to employ a well mixed reactor which guarantees uniform reactant concentration in the entire volume or in the cross sections of the reactor. The balances realized for the large-scale reactor are solved simultaneously, optimized and validated with the experimental results. ${ }^{[6]}$

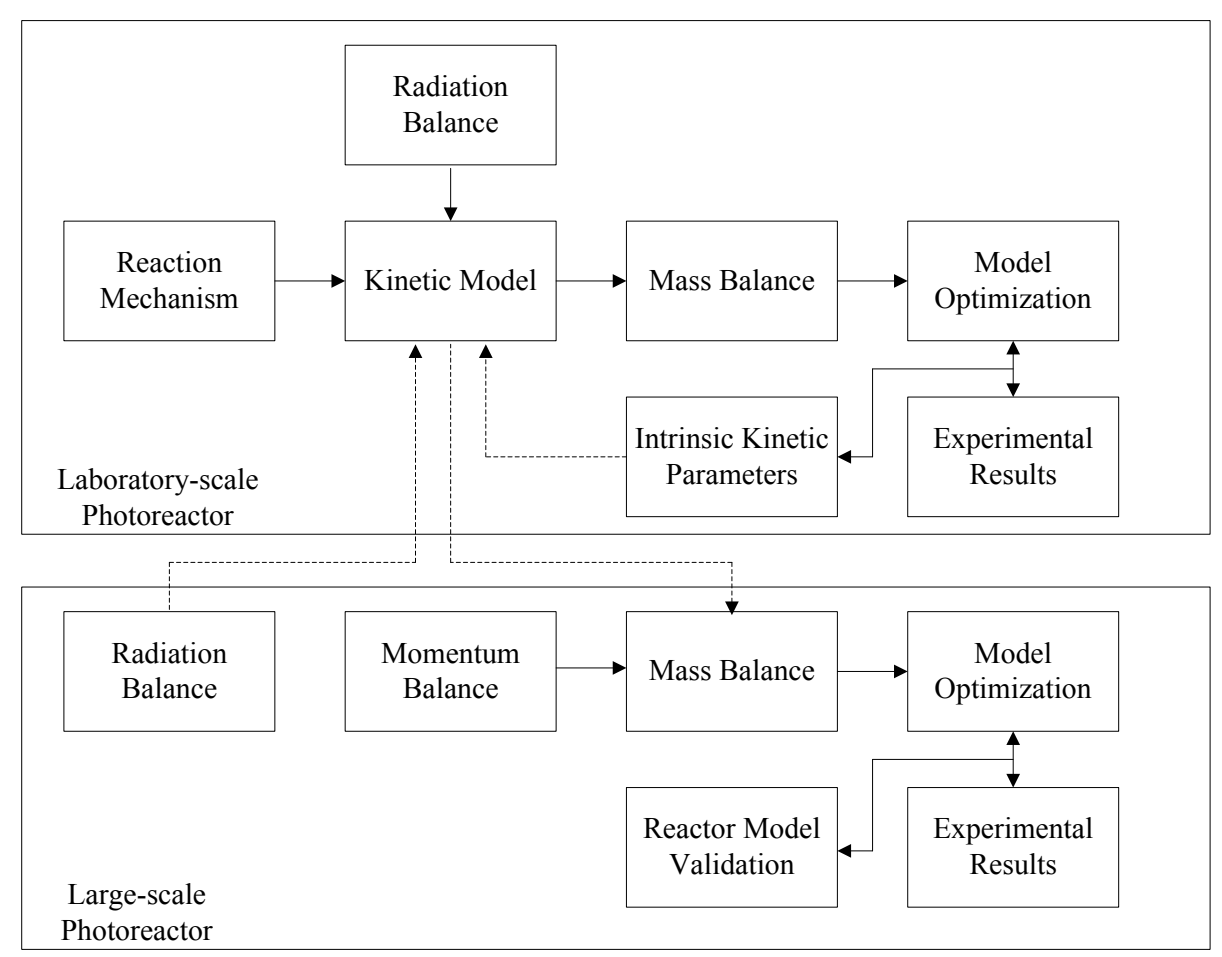

Fig. 1.1.Scale-up methodology of photoreactors (Adapted from Ghafoori ${ }^{[6]}$ ).

The following aspects need to be considered when applying this method: ${ }^{[8]}$

- a validated reaction scheme is required

- operation of the laboratory-scale reactor with no mass transfer limitations

- the same catalyst properties (preparation protocol and morphology) in the case of heterogeneous catalytic system

- isothermal conditions

- light sources with the same spectral radiation output 
- application and rigorously solving the mathematical model for the laboratory and large scale reactors

Applications of this scale-up methodology are briefly described in section 1.7.

\subsection{Kinetic model}

The existence of the radiation-activated step distinguishes a photochemical from a thermal reaction. ${ }^{[9]}$ In a single photon absorption photochemical reaction (which we define here to include both photosensitized and photocatalytic reactions), the rate of this initiation step (primary event), $r$, is proportional to the local volumetric rate of photon absorption (LVRPA): ${ }^{[10,11]}$

$$
r=\Phi_{\lambda} e_{\lambda}^{a}
$$

where $\Phi$ is the reaction quantum yield, $e^{a}$ is the local volumetric rate of photon absorption. Both properties are defined at a given wavelength, $\lambda$, and for a given species. ${ }^{[11]}$

The LVRPA is dependent on the radiation field in the reactor and can be determined by solving the radiation balance which will be described in section $1.4 .^{[12]}$

The reaction quantum yield in this case is called primary quantum yield ${ }^{[10]}$ A primary reaction includes the absorption of the photon which leads to the formation of an excited state followed by the formation of an intermediate or final product and/or deactivation processes (e.g., non-radiative, radiative: fluorescence, phosphorescence). ${ }^{[13]}$ However, it excludes the thermal reactions of the intermediate or product which may take place in the reactor space. As the primary quantum yield considers a single photon absorption process, its value will be comprised between 0 and 1 . A value of 0 will indicate that only deactivation processes occur and a value of 1 that no deactivation processes are present (quantitative conversion of the excited state molecules to the product). In some cases, the quantum yield is reported to be higher than 1 (e.g. photochemical polymerization reactions). However, this measure represents the overall quantum yield and considers both photochemical and thermal reactions. ${ }^{[13]}$ Care must be taken when using this value, as it does not have the quality of an intrinsic kinetic property. ${ }^{[10]}$

In heterogeneous catalytic systems it is difficult to rigorously determine the number of absorbed photons, because a large amount of photons is lost through scattering. Therefore, the quantum yield can be determined only after quantifying the scattering effects (a method to determine the optical properties of suspended catalyst is described by Alfano et al. in chapter 4). However, one needs to pay attention because, most of the investigations report the apparent quantum yield and not the "true" quantum yield. The former property is defined as the ratio of the rate of the reactant consumed or product formed and amount of incident photons. ${ }^{[14]}$

Establishing the kinetic model is a demanding process especially in the case of reactions with complex mechanism. As a validated kinetic model is required, the scale-up investigations are generally based on extensive kinetic studies of the reactions to be implemented at large-scale. ${ }^{[6,15-18]}$

\subsection{Radiation balance}

\subsubsection{Radiative Transfer Equation}

The requirement of LVRPA determination leads to the coupling of the photochemical reaction rate with the radiation balance. This property can be calculated from the radiation intensity integrated over all propagation directions as in Eq. $2 .^{[12,19]}$ 


$$
e^{a}=\int_{\lambda_{1}}^{\lambda_{2}} \kappa_{\lambda}(\boldsymbol{x}) \int_{\Omega=4 \pi} I_{\lambda, \mathbf{\Omega}}(\boldsymbol{x}) d \Omega d \lambda
$$

where $\kappa_{\lambda}$ is the volumetric absorption coefficient, $I_{\lambda, \Omega}$ is the spectral intensity (reported also as specific spectral intensity), $\lambda$ is the radiation wavelength, $\boldsymbol{x}$ is a position vector in a $3 \mathrm{D}$ space, $\Omega$ is the solid angle of radiation propagation, $\boldsymbol{\Omega}$ is the unit vector in the direction of radiation propagation.

The spectral intensity can be obtained by solving the equation which describes the transport of radiation through the reacting medium (Radiative Transport Equation (RTE)). By assuming negligible radiation emission due to the low operating temperature, steady state, elastic scattering (i.e., scattering changes only the direction of the photons), multiple and independent scattering, the RTE can be expressed as: ${ }^{[12,19]}$

$$
\frac{d I_{\lambda, \boldsymbol{\Omega}}(\boldsymbol{x})}{d s}=-\kappa_{\lambda}(\boldsymbol{x}) I_{\lambda, \boldsymbol{\Omega}}(\boldsymbol{x})-\sigma_{\lambda}(\boldsymbol{x}) I_{\lambda, \boldsymbol{\Omega}}(\boldsymbol{x})+\frac{\sigma_{\lambda}(\boldsymbol{x})}{4 \pi} \int_{\Omega^{\prime}=4 \pi} p\left(\boldsymbol{\Omega}^{\prime} \rightarrow \boldsymbol{\Omega}\right) I_{\lambda, \mathbf{\Omega}^{\prime}}(\boldsymbol{x}) d \boldsymbol{\Omega}^{\prime}
$$

where $\sigma_{\lambda}$ is the volumetric scattering coefficient, $p\left(\boldsymbol{\Omega}^{\prime} \rightarrow \boldsymbol{\Omega}\right)$ is the scattering phase function, $\Omega^{\prime}$ is the new solid angle of radiation propagation after scattering, $s$ is the linear coordinate along the direction of radiation propagation $\boldsymbol{\Omega}$.

The RTE is an integro-differential equation, where the first term on the right hand side quantifies the loss of radiation through absorption, the second term, the loss of radiation due to out-scattering and the third term the radiation gained as a consequence of in-scattering (resulting from multiple scattering phenomena). ${ }^{[12]}$ The equation has to be solved for each wavelength, $\lambda$, of the discretized wavelength range employed during the photochemical reaction. ${ }^{[19]}$ A detailed description of the parameters involved in the RTE is offered by Alfano et al. in chapter 4.

\subsubsection{Numerical resolution of radiative transport equation}

The RTE is simplified to the Bouguer-Lambert-Beer law when scattering is negligible i.e. the radiation travels through a homogeneous medium. This case is described in more detail by $\mathrm{Su}$ et al. in chapter 2 . However, it is difficult to analytically solve the equation when it is applied to heterogeneous systems. Solving the RTE involves appropriate boundary conditions and numerical methods. The boundary conditions for the RTE depend on the type of emission of the lamp and the geometrical properties of the lamp-reactor system. ${ }^{[10]}$ Lamp emission models were reviewed previously ${ }^{[9,20]}$ and will not be discussed in this chapter. The RTE solution can be calculated using discretization, such as the DiscreteOrdinate Method, by probabilistic numerical methods such as Monte Carlo or by simplified radiation field models, such as Two-Flux and Six-Flux Methods. ${ }^{[19,21]}$ The main methods used for solving the RTE will be briefly introduced from the most rigorous to the most simplified methods.

The Discrete-Ordinate Method (DOM) employs a directional and spatial discretization which transforms the RTE into a system of algebraic equations. ${ }^{[22]}$ These equations describe the transport of photons in such a way that they can be solved following the direction of propagation, starting from the values provided by the boundary conditions. ${ }^{[19]}$ The DOM is recognized as one of the most accurate methods, because it does not involve any assumption, other than the discretization of pertinent partial different equations. ${ }^{[23,24]}$ However, the application of the DOM for its solution requires a high degree of numerical accuracy, which is reflected in a high computational time. ${ }^{[25]}$ As the DOM does not guarantee the conservation of the radiant energy at the surfaces of complex geometries especially for anisotropic scattering, a variant DO model based on the Finite-Volume Method (FVM) was developed. FVM allows 
to conserve radiative energy, as the RTE is integrated over both the control angle and the control volume, unlike the DOM, in which the RTE is integrated over the control volume only. ${ }^{[2]}$

The Monte Carlo method (MC) is based on the probabilistic behavior of photons, since they are emitted from the light source until their absorption or escape through the reactor wall. ${ }^{[2]}$ Firstly, the position and the direction of the photons are randomly determined taking into account the geometry (e.g., the position of the light source). Then, the photons travel through the medium and can be absorbed or scattered in a certain direction. The probabilities of being absorbed or scattered depend on the absorption and scattering coefficients. For the scattered photon, a new direction is determined whose probability depends on the phase function. ${ }^{[13]}$ The MC method has the advantage that it is relatively simple to apply, especially for complex geometries. ${ }^{[26]}$ As it can be observed in Table 1.1, MC simulations were successfully applied to annular reactors, monolith reactors, multiplate reactors etc. Moreover, this method is considered to be precise, as no approximations are included regarding the radiation field; its precision is determined by the number of generated photons and their stochastic behavior. As a consequence, the MC method can easily become computationally expensive. ${ }^{[20,27]}$

The Two-Flux Method (TFM) does not use the RTE in an explicit manner. The incident radiation is divided into two opposite directions and implies two coupled ordinary differential equations for which analytical solutions can be easily determined. ${ }^{[20]}$ It is based on the assumption that the scattered photons are scattered only in backward direction. It is probably the simplest method to study the light intensity distribution in heterogeneous systems. ${ }^{[20]}$ The Six-Flux Method (SFM) is a three-dimensional extension of the TFM, so the photons are scattered in six principal directions with respect to the incoming radiation. ${ }^{[28]}$ Its mathematical structure is of algebraic nature. Therefore, its implementation in reactors of different scales and radiation sources implies low complexity numeric procedures and short computational times. ${ }^{[25]}$ Moreover, the SFM has the advantage of offering a quite accurate representation of the radiation field while using simple modeling equations. ${ }^{[29,30]}$

In the following we highlight the difference in results obtained for different levels of model complexity. Li Puma and Brucato compared the relative error between experimental results and three models, namely Lambert-Beer, TFM and SFM. ${ }^{[21]}$ The experimental results were acquired for isoproturon oxidation in aqueous suspensions of $\mathrm{TiO}_{2}$ in an annular photocatalytic reactor operated in recirculation mode. The Lambert-Beer model neglects scattering within the reactor space, therefore it overestimates the amount of radiation available for the photoreaction. As it can be observed in Fig. 1.2, the isoproturon conversion simulated using the Lambert-Beer model is higher than the conversion achieved in the experiments. On the other hand, the TFM considers back-scattering only (no contribution from inscattering phenomena). Consequently, the results predicted by TFM underestimate the conversion of the herbicide. The study of Li Puma and Brucato clearly shows that a) the experimental results will be between the results predicted by the two models, Beer-Lambert and TFM; b) the radiation scattering should be considered in order to avoid the erroneous determination of the kinetic parameters, which are essential for the scale-up studies. ${ }^{[21]}$ 


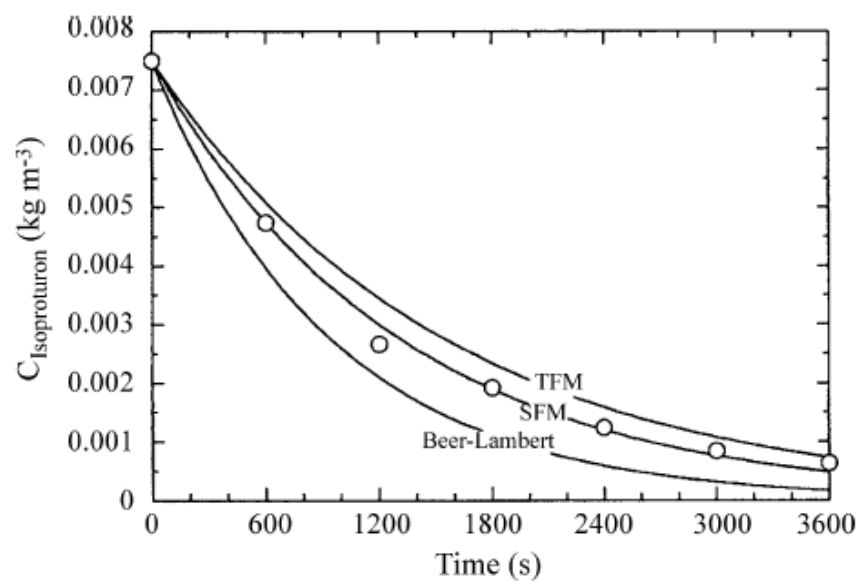

Fig. 1.2. Modeling the photocatalytic oxidation of isoproturon using Beer-Lambert model, TFM and SFM. Lines are predicted results and circles the experimental results ${ }^{[21]}$ (Reprinted with permission of ${ }^{C} 2007$ Elsevier B.V.).

For further examples of applications of the different modeling strategies to various reactor geometries the reader is referred to the selected references in Table 1.1.

Table 1.1. Applications of the main methods used for RTE resolution.

\begin{tabular}{|c|c|c|}
\hline Method & Reactor configuration & Reference \\
\hline \multirow[t]{4}{*}{$\overline{\mathrm{DOM}}$} & Annular & {$[23]$} \\
\hline & & [31] \\
\hline & Cylindrical, rectangular & [5] \\
\hline & Cylindrical & [19] \\
\hline \multirow[t]{2}{*}{$\overline{\mathrm{FVM}}$} & Annular & [24] \\
\hline & Single and multiple-lamp reactors & [32] \\
\hline \multirow[t]{9}{*}{$\overline{\mathrm{MC}}$} & Annular & [33] \\
\hline & & [34] \\
\hline & & [35] \\
\hline & & [36] \\
\hline & & [37] \\
\hline & Plane-slab & [29] \\
\hline & Monolyth & [38] \\
\hline & Multi-plate & [26] \\
\hline & Cylindrical & [39] \\
\hline \multirow[t]{6}{*}{$\overline{\mathrm{TFM}}$} & Plane-slab & [29] \\
\hline & Annular & [21] \\
\hline & Thorus-shaped photobioreactor & [40] \\
\hline & Flat-plate & [41] \\
\hline & Microreactor & [13] \\
\hline & Tubular & [25] \\
\hline \multirow[t]{8}{*}{$\overline{\text { SFM }}$} & Annular & [28] \\
\hline & & [21] \\
\hline & & [7] \\
\hline & & [30] \\
\hline & Plane-slab & [29] \\
\hline & Falling film & [7] \\
\hline & $\begin{array}{l}\text { Compound parabolic } \\
\text { Collectors (CPC) }\end{array}$ & [42] \\
\hline & Tubular & [25] \\
\hline
\end{tabular}




\subsection{Mass balance}

The mass balance for a component $i$ in a reactor considering non-steady-state is: ${ }^{[43]}$

$$
\frac{\partial C_{i}}{\partial t}=-\left(\nabla \cdot C_{i} \mathbf{v}\right)-\left(\nabla \cdot J_{i}\right)+R_{i}
$$

where $C_{i}$ is the molar concentration of species $i, \mathbf{v}$ is the velocity vector of the fluid, $J_{i}$ is the molar diffusion flux and $R_{i}$ is the volumetric rate of generation of component $i$. The kinetic model which includes the LVRPA is incorporated in the mass balance through the reaction rate $R_{i}$.

$\partial C_{i} / \partial t$ represents the rate of increase of component $i$ per unit volume, $\left(\nabla \cdot C_{i} \mathbf{v}\right)$ is the net rate of addition of $i$ per unit volume by convection, $\left(\nabla \cdot J_{i}\right)$ is the net rate of addition of $i$ per volume by diffusion and $R_{i}$ is the net rate of production of $i$ per unit volume by reaction. ${ }^{[43]}$ According to Fick's law, the diffusive flux for a constant diffusivity of component $i, D_{i}$, is: ${ }^{[43]}$

$$
J_{i}=-D_{i}\left(\nabla C_{i}\right)
$$

and the mass balance for component $i$ becomes: ${ }^{[43]}$

$$
\frac{\partial C_{i}}{\partial t}=-\left(\nabla \cdot C_{i} \mathbf{v}\right)+D_{i}\left(\nabla^{2} \cdot C_{i}\right)+R_{i}
$$

The mass balance can be expressed in rectangular coordinates (to be used in the case of flat plate reactors, for example):

$$
\frac{\partial C_{i}}{\partial t}=-\left(v_{x} \frac{\partial C_{i}}{\partial x}+v_{y} \frac{\partial C_{i}}{\partial y}+v_{z} \frac{\partial C_{i}}{\partial z}\right)+D_{i}\left(\frac{\partial^{2} C_{i}}{\partial x^{2}}+\frac{\partial^{2} C_{i}}{\partial y^{2}}+\frac{\partial^{2} C_{i}}{\partial z^{2}}\right)+R_{i}
$$

or in cylindrical coordinates (for annular and cylindrical reactors):

$$
\frac{\partial C_{i}}{\partial t}=-\left(v_{r} \frac{\partial C_{i}}{\partial r}+v_{\theta} \frac{1}{r} \frac{\partial C_{i}}{\partial \theta}+v_{z} \frac{\partial C_{i}}{\partial z}\right)+D_{i}\left(\frac{1}{r} \frac{\partial}{\partial r}\left(r \frac{\partial C_{i}}{\partial r}\right)+\frac{1}{r^{2}} \frac{\partial^{2} C_{i}}{\partial \theta^{2}}+\frac{\partial^{2} C_{i}}{\partial z^{2}}\right)+R_{i}
$$

In the following subsections, we will show possible simplifications of Eqs. 7 and 8 in various cases. The material balance will be discussed for laboratory-scale reactors where it was used for determining the intrinsic kinetic parameters and for large scale reactors where it provided the tool to predict the reactor performance.

\subsubsection{Mass balance in laboratory scale reactor}

The general approach in kinetic investigations is to bring the studied system in the reaction rate limited regime, so the apparent reaction rate can reach the intrinsic value. ${ }^{[4]}$ The mass balance is used to predict the conversion of a species, $i$, which is then compared with the experimental measurements. The unknown intrinsic kinetic parameters are obtained with a non-linear parameter estimator and an optimization program. The final outcome is a kinetic model with known constants that can be used for scale-up purposes and reactor optimization, as this result is independent of the employed light source, particular reactor geometry and operating conditions. ${ }^{[12]}$ 


\subsubsection{Mass balance in batch reactor with recirculation}

The classical experimental set-up used for kinetic studies is composed of a laboratory-scale reactor and a mixing tank. In the following part we describe possible simplifications of the general mass balance and in which conditions they can be considered.

Labas studied the kinetics of oxidation of dilute aqueous formic acid solutions with UV radiation and $\mathrm{H}_{2} \mathrm{O}_{2}{ }^{[45]}$ The kinetic parameters were obtained in a well-stirred, small, batch flat-plate photoreactor. The authors considered the following conditions:

- the entire system operates under well-stirred conditions (well mixed)

- the ratio of the reactor volume $\left(70 \mathrm{~cm}^{3}\right)$ to the tank volume $\left(2000 \mathrm{~cm}^{3}\right)$ is $<1$ (recommended to be $<<1$ )

- the recirculating flow rate is high in order to have differential conversion per pass in the photoreactor

Since the reactor content was well mixed, the net rate of addition of $i$ per unit volume by convection and by diffusion was assumed negligible and the mass balance could be written as:

$$
\frac{\partial C_{i}(t)}{\partial t}=R_{i, \lambda}
$$

Equation 9 was then integrated over the system volume. The integral of the left hand-side could be divided in two volumes: the photoreactor volume $\left(V_{R}\right)$ and the remaining system volume $\left(V_{T}-V_{R}\right.$, where $\mathrm{V}_{\mathrm{T}}$ is the total volume). The reaction is considered to only take place in the reactor, which yields:

$$
\int_{V_{R}} \frac{\partial C_{i}(t)}{\partial t} d V+\int_{V_{T}-V_{R}} \frac{\partial C_{i}(t)}{\partial t} d V=\int_{V_{R}} R_{i, \lambda} d V
$$

As the volumes are constant, the derivative and integral signs can be interchanged. To each resulting term, the volume-average theorem is applied with \langle\rangle indicating the volume-averaged value.

$$
V_{R} \frac{\partial}{\partial t}\left\langle C_{i}(t)\right\rangle_{V_{R}}+\left(V_{T}-V_{R}\right) \frac{\partial}{\partial t}\left\langle C_{i}(t)\right\rangle_{V_{T}-V_{R}}=V_{R}\left\langle R_{i, \lambda}(x, t)\right\rangle_{V_{R}}
$$

Equation 11 can be divided by $\mathrm{V}_{\mathrm{T}}$ and rearranged as follows:

$$
\frac{V_{R}}{V_{T}} \frac{\partial}{\partial t}\left[\left\langle C_{i}(t)\right\rangle_{V_{R}}-\left\langle C_{i}(t)\right\rangle_{V_{T}-V_{R}}\right]+\frac{\partial}{\partial t}\left\langle C_{i}(t)\right\rangle_{V_{T}-V_{R}}=\frac{V_{R}}{V_{T}}\left\langle R_{i, \lambda}(x, t)\right\rangle_{V_{R}}
$$

Because $V_{R} / V_{T}$ was small $\left(V_{R}=70 \mathrm{~cm}^{3}\right.$ and $\left.V_{T}=2000 \mathrm{~cm}^{3}\right)$ and the conversion per pass is differential (difference between outlet and inlet concentrations in the reactor was small) the first term of Eq. 12 was considered negligible. Consequently, when the system was well-mixed, the change in concentrations in $V_{T}-V_{R}$ was equal to the changes in concentrations in the tank as written in Eq. 13:

$$
\left.\frac{\mathrm{d} C_{i}(t)}{\mathrm{d} t}\right|_{\text {Tank }}=\frac{V_{R}}{V_{T}}\left\langle R_{i, \lambda}(x, t)\right\rangle_{V_{R}}
$$

The reaction rate averaged over the reactor volume was estimated using the kinetic model and then compared with the reaction rate determined experimentally (evolution of formic acid concentration with time). The reaction rate was a function of position $(x)$ because of the light distribution inside the reaction space. The kinetic parameters obtained in the described system were applied in the modeling of a large scale reactor. The predicted concentrations were in reasonably good agreement with the measured concentrations. The errors found when running the large-scale reactor under different conditions were comprised between 6.6 and $11.8 \%{ }^{[45]}$ 
In kinetic studies the predominantly used photoreactor geometries which are operated in recirculation regime are the annular reactor ${ }^{[6,7,30,46]}$, flat-plate reactor ${ }^{[16,45,47-50]}$ and cylindrical reactor ${ }^{[5,19,51]}$.

\subsubsection{Mass balance in microreactors}

Recently, microreactors have been used for the determination of the kinetic constants of photocatalytic reactions. ${ }^{[4,52-54]}$ Microreactors offer the advantage of a higher spatial illumination homogeneity and better light penetration through the reactor depth in comparison to other laboratory-scale reactors which are generally employed for kinetic studies.

When considering the mass balance for microreactors, usually a plug flow reactor model in the absence or presence of mass transport limitations is assumed. The mass balance for a plug flow reactor in the presence of radial concentration profiles and axial dispersion in two-dimensional rectangular coordinates $(x, y)$ can be expressed as (the channel length corresponds to the $x$-direction and the channel width to the y-direction):

$$
v_{x} \frac{d C_{i}}{d x}-D_{i} \frac{d^{2} C_{i}}{d y^{2}}=-R_{i}
$$

Equation 14 is obtained from Equation 7 by considering the following assumptions: ${ }^{[13,43]}$

- $\quad$ steady state $\left(\partial \mathrm{C}_{\mathrm{i}} / \partial \mathrm{t}=0\right)$

- unidirectional flow ( $v_{x}$ is the non-zero velocity component, $v_{y}=v_{z}=0$ )

- axial diffusion negligible compared to axial convection $\left(D_{i} \partial^{2} C_{i} / \partial x^{2} \approx 0\right)$

- fully developed flow ( $v_{x}$ does not change in axial direction)

- isothermal conditions (constant fluid properties)

Charles investigated the kinetics of photocatalytic degradation of salicylic acid in microreactors (1$2 \mathrm{~mm}$ channel diameter) with immobilized titanium dioxide as photocatalyst. ${ }^{[53]}$ Their results highlight the importance of considering external mass transfer limitations and the influence of axial dispersion in photoreactor modeling for determining the kinetic constants in microreactors.

Firstly, the kinetic parameters were determined by fitting the predicted results using the mass balance equation under the assumption of perfect plug-flow with the experimental results acquired under different residence times. In Fig. 1.3a, the experimental results are illustrated with symbols and the predictions are illustrated by lines. The continuous line was calculated with the rate constants $\left(k_{a p p}, K_{a p p}\right)$ fitted at $4 \mathrm{~mL} / \mathrm{h}$ flow rate, the dashed line with the parameters fitted at $10 \mathrm{~mL} / \mathrm{h}$ and the dotted line with the parameters fitted at $20 \mathrm{~mL} / \mathrm{h}$. As it can be observed in Fig. 1.3a, the obtained apparent kinetic constants $k_{a p p}$ and $K_{a p p}$ vary with the flow rate and neither set of constants was able to match the degradation rate at all flow rates. 

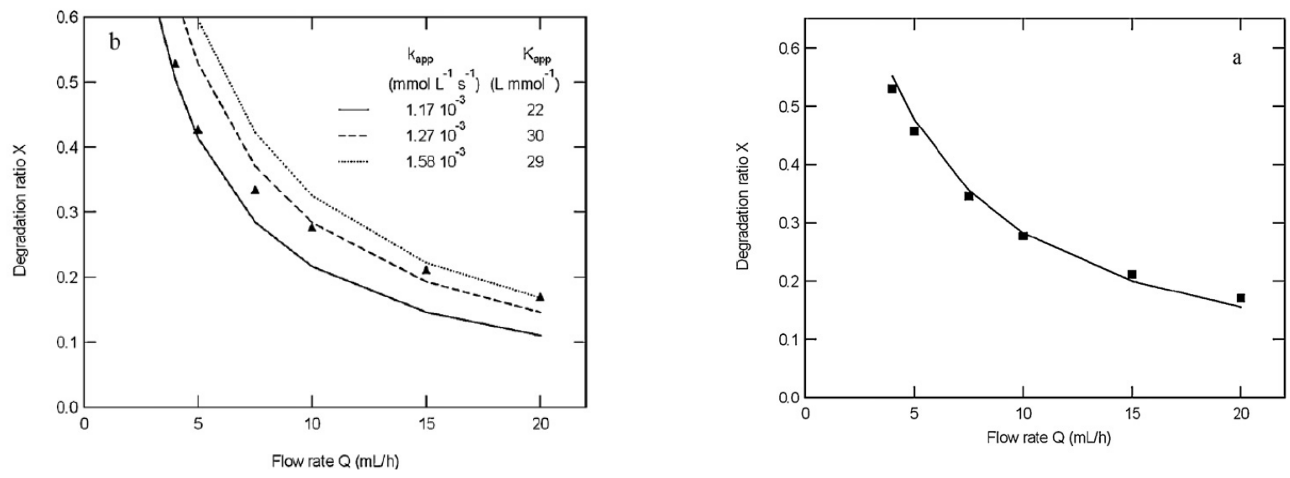

Fig.1.3. The degradation ratio of salicylic acid (X) as a function of flow rate (Q). a) Experimental degradation ratios (symbols) and calculated degradation ratios assuming a perfect plug-flow reactor (lines); The continuous line was calculated with the rate constants $\left(k_{\text {app }}, K_{\text {app }}\right)$ fitted at $4 \mathrm{~mL} / \mathrm{h}$ flow rate, the dashed line with the parameters fitted at $10 \mathrm{~mL} / \mathrm{h}$ and the dotted line with the parameters fitted at $20 \mathrm{~mL} / \mathrm{h}$; b) Experimental degradation ratios (symbols) and calculated degradation ratios assuming a plug flow reactor with mass transport limitations (line). Fitting parameters are $k=1.70 \cdot 10^{-3} \mathrm{mmol} \mathrm{L}^{-1} \mathrm{~s}^{-1}$ and $K=24 \mathrm{~L} \mathrm{mmol}^{-1}$. All shown experimental results were acquired using a light source with $I_{\text {incident }}=1.5 \mathrm{~mW} / \mathrm{cm}^{2}$ and a microreactor with $d_{\text {channel }}=$ $2 \mathrm{~mm} .{ }^{[53]}$ (Reprinted with permission of (C) 2011 Elsevier B.V.).

In a second case, Charles considered a model based on the plug flow reactor assumption with convective mass transport and axial diffusion. ${ }^{[53]}$ As it can be seen in Fig. 1.3b, the fitted kinetic parameters $\mathrm{k}$ $\left(1.70 \cdot 10^{-3} \mathrm{mmolL}^{-1} \mathrm{~s}^{-1}\right)$ and $\mathrm{K}\left(24 \mathrm{Lmmol}^{-1}\right)$ were able to predict the degradation of salicylic acid at all flow rates. This shows that the obtained kinetic parameters are independent of the operating conditions. However, the rate constant $\mathrm{k}$ was found to be significantly dependent on the light intensity, as the radiance balance was not considered when developing the kinetic model.

Aillet conducted kinetic investigations of a photochemical reaction in a spiral-shaped microreactor (0.5 mm inner diameter) irradiated by an ultraviolet/light-emitting diode array. ${ }^{[54]} \mathrm{A}$ T-photochromic system was studied which involves a reversible reaction between a closed form and an open form. The kinetic parameters of this reaction were determined using the modeling tools previously developed by the group. ${ }^{[13]}$ The employed model used the mass balance as expressed in Eq. 14. In addition to the previously discussed study by Charles ${ }^{[53]}$, the effect of the radiation intensity was considered in the reaction rate through the LVRPA. Therefore, the determined kinetic parameters were independent of the geometry, operating conditions and light source. This is proved in Fig. 1.4 where the variation of absorbance, $\mathrm{A}_{\mathrm{obs}}$, (the production of the open form product can be determined from the measured absorbance at $610 \mathrm{~nm}$ ) with the residence time, $\tau_{\mathrm{s}}$, is illustrated. The symbols represent the experimental data and the dashed lines are the model predictions with the fitted kinetic parameters. As it can be observed, the determined kinetic parameters are able to predict the conversion variation with the residence time (flow rate) and with the photon flux, $q_{p}$. 


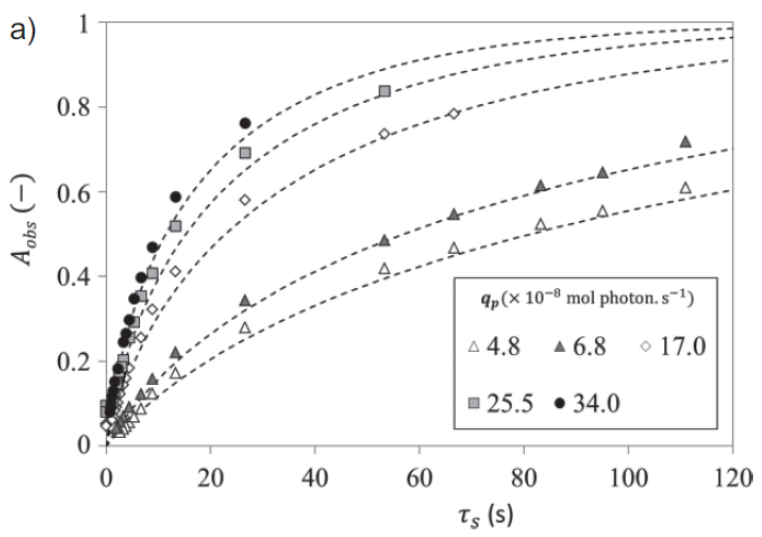

Fig.1.4. The variation of the monitored absorbance $\left(\mathrm{A}_{\mathrm{obs}}\right)$ at $610 \mathrm{~nm}$ with the residence time $\left(\tau_{\mathrm{s}}\right)$ at different photon fluxes $\left(q_{p}\right)$. Comparison between experimental (symbols) and predicted absorbance (dotted lines). ${ }^{[54]}$ (Reprinted with permission of ${ }^{C}$ 2016 WILEY-VCH Verlag GmbH \& Co. KGaA, Weinheim )

\subsubsection{Mass balance in large-scale reactors}

The kinetic parameters estimated using a laboratory-scale reactor can be applied in the kinetic model, which in turn can then be included in the mass balance of a large-scale reactor.

Marugán developed a model of a bench-scale, annular reactor for the photocatalytic disinfection of water (inactivation of Escherichia coli bacteria). ${ }^{[55]}$ The reactor was operated under continuous flow with no perfect mixing.

The mass balance as expressed in Eq. 8 was applied to the annular reactor under the following assumptions:

- $\quad$ steady state $\left(\partial \mathrm{C}_{\mathrm{i}} / \partial \mathrm{t}=0\right)$

- unidirectional axial flow ( $v_{z}$ is the non-zero velocity component, $v_{r}=v_{\theta}=0$ )

- $\quad$ azimuthal symmetry $(\partial / \partial \theta=0)$

- negligible axial diffusion compared to the convective flux in the axial direction $\left(D_{i} \partial^{2} C_{i} / \partial z^{2} \approx 0\right)$

- fully developed flow ( $v_{x}$ does not change in axial direction, $v_{x}(\mathrm{r})$ )

- isothermal conditions (constant fluid properties)

The resulting mass balance expression in cylindrical coordinates is:

$$
v_{z} \frac{\partial C_{i}}{\partial z}=D_{i}\left(\frac{1}{r} \frac{\partial}{\partial r}\left(r \frac{\partial C_{i}}{\partial r}\right)\right)+R_{i}
$$

Figure 1.5 illustrates the variation of bacteria concentration expressed in colony forming units (CFU) with the catalyst loading after 2000s of irradiation. Comparing the experimental results (symbols) with the predicted data (line), it can be observed that the photoreactor model based on Eq. 15 was able to predict an optimal catalyst concentration in the range of $0.1-0.2 \cdot 10^{-3} \mathrm{gcm}^{-3}$ catalyst concentration.

This optimum catalyst concentration range was not observed in the experiments which were carried out in the well mixed laboratory-scale reactor for determining the kinetic parameters. The reduced activity observed in the presence of high catalyst loadings indicates the occurrence of mass transport limitations and should only appear in the non-perfectly mixed photoreactors systems. 


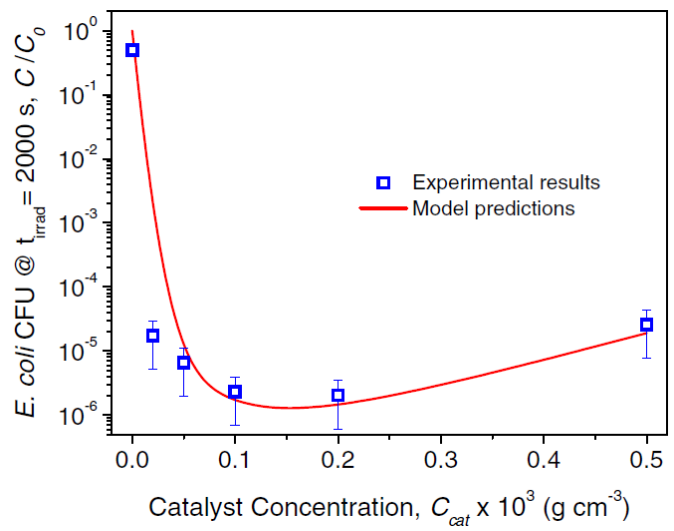

Fig.1.5. Bacteria concentration after $2000 \mathrm{~s}$ as a function of catalyst concentration. Comparison between experimental results (symbols) and model predictions (line) ${ }^{[55]}$ (Reprinted with permission of (C 2012 Elsevier B.V.)

\subsection{Momentum balance}

\subsubsection{Conservation of momentum}

The velocity profile which has to be included in the mass balance can be determined from the momentum balance. The Cauchy momentum equation is a general statement of conservation of linear momentum. It provides the starting point for analyzing both Newtonian and non-Newtonian flows for either constant and variable density: ${ }^{[56]}$

$$
\frac{\partial \rho \mathbf{v}}{\partial t}=-\nabla \cdot(\rho \mathbf{v v})-\nabla P-\nabla \cdot \tau+\rho \mathbf{g}
$$

where $\rho$ is the mass density, $\mathbf{v}$ is velocity vector, $t$ is time, $P$ is pressure, $\boldsymbol{\tau}$ is the viscous stress tensor, $\mathbf{g}$ is the gravitational acceleration vector.

$\partial \rho \mathbf{v} / \partial t$ represents the rate of increase of momentum per unit volume, $\nabla \cdot(\rho \mathbf{v v})$ is the rate of momentum addition by convection per unit volume, $\nabla P$ and $\nabla \cdot \tau$ represent the rate of momentum addition by molecular transport per unit volume, $\rho \mathbf{g}$ is the external force on fluid per unit volume. ${ }^{[57]}$

Considering Newtonian fluids with constant density (incompressible fluids), constant viscosity and inserting the Newtonian expression of $\boldsymbol{\tau}$ in the previous equation results in the Navier-Stokes Equations: ${ }^{[56]}$

$$
\rho\left(\frac{\partial \mathbf{v}}{\partial t}+\mathbf{v} \cdot \nabla \mathbf{v}\right)=-\nabla P+\mu \nabla^{2} \mathbf{v}+\rho \mathbf{g}
$$

Together with the continuity equation (assuming constant density):

$$
\nabla \cdot \mathbf{v}=0
$$

the Navier-Stokes Equations allow to analyze the flow of liquids or gases at moderate velocities. ${ }^{[56]}$

The Navier-Stokes Equations can be simplified depending on the considered situation, and in the following we will describe the simplifications for parallel-plate channel. This case is often encountered 
when describing flat-plate photoreactors. We start by considering the Navier-Stokes Equations in component form in rectangular coordinates. ${ }^{[56]}$

$$
\begin{aligned}
& \rho\left(\frac{\partial v_{x}}{\partial t}+v_{x} \frac{\partial v_{x}}{\partial x}+v_{y} \frac{\partial v_{x}}{\partial y}+v_{z} \frac{\partial v_{x}}{\partial z}\right)=-\frac{\partial P}{\partial x}+\mu\left(\frac{\partial^{2} v_{x}}{\partial x^{2}}+\frac{\partial^{2} v_{x}}{\partial y^{2}}+\frac{\partial^{2} v_{x}}{\partial z^{2}}\right)+\rho g_{x} \\
& \rho\left(\frac{\partial v_{y}}{\partial t}+v_{x} \frac{\partial v_{y}}{\partial x}+v_{y} \frac{\partial v_{y}}{\partial y}+v_{z} \frac{\partial v_{x}}{\partial z}\right)=-\frac{\partial P}{\partial y}+\mu\left(\frac{\partial^{2} v_{y}}{\partial x^{2}}+\frac{\partial^{2} v_{y}}{\partial y^{2}}+\frac{\partial^{2} v_{y}}{\partial z^{2}}\right)+\rho g_{y} \\
& \rho\left(\frac{\partial v_{z}}{\partial t}+v_{x} \frac{\partial v_{z}}{\partial x}+v_{y} \frac{\partial v_{z}}{\partial y}+v_{z} \frac{\partial v_{z}}{\partial z}\right)=-\frac{\partial P}{\partial z}+\mu\left(\frac{\partial^{2} v_{z}}{\partial x^{2}}+\frac{\partial^{2} v_{z}}{\partial y^{2}}+\frac{\partial^{2} v_{z}}{\partial z^{2}}\right)+\rho g_{z}
\end{aligned}
$$

Next, the following assumptions are considered:

- $\quad$ steady state $(\partial / \partial \mathrm{t}=0)$

- unidirectional axial flow ( $v_{x}$ is the non-zero velocity component, $v_{y}=v_{z}=0$; consequently, all terms in Eqs. 20 and 21 vanish)

- fully developed flow $\left(\partial v_{x} / \partial x=0\right.$, then it also follows that $\left.\partial^{2} v_{x} / \partial x^{2}=0\right)$

- the $z$ dimension is large enough that edge effects are negligible, which results in $v_{x}=v_{x}(y)$

- no external force (e.g., the channel is placed horizontally, therefore $\rho g_{x}=0$ )

and the simplified equation can be written as:

$$
0=-\frac{\partial P}{\partial x}+\mu\left(\frac{\partial^{2} v_{x}}{\partial y^{2}}\right)
$$

By integrating Eq. 22, the velocity profile in the parallel-plate channel can be obtained.

\subsubsection{Velocity profile in common photoreactor configurations}

The expressions for describing the velocity profile for the most encountered cases in photoreactor modeling are shown together with their applications reported in literature.

The velocity profile, $v_{x}(y)$, of a steady-state, fully developed laminar flow in parallel-plate channels can be expressed in rectangular coordinates as: ${ }^{[47]}$

$$
\begin{gathered}
v_{x}(y)=v_{\max }\left[1-\left(\frac{2 y}{H}-1\right)^{2}\right] \\
v_{\max }=\frac{3}{2}\left\langle v_{x}\right\rangle
\end{gathered}
$$

where $v_{\max }$ is the maximum fluid velocity in the channel, $\left\langle v_{x}\right\rangle$ is the mean fluid velocity in the channel, $y$ is the rectangular coordinate, $H$ is the height of the channel. The flow representation for the considered parallel-plate channel is illustrated in Fig.1.6a.

a)

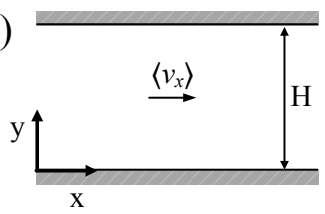

b)

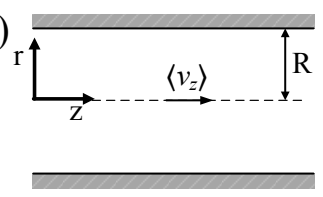

c)

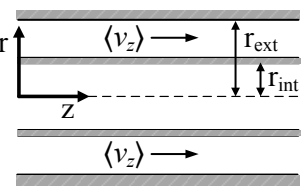

Fig. 1.6. Representation of the flow in a) a parallel-plate channel; b) a tubular channel; c) an annular reactor. 
Equation 23 was used under slightly different form in the modeling of flat-plate photoreactors ${ }^{[43,47]}$ and photomicroreactors. $^{[13,44]}$

The expression for the velocity profile $v_{x}(r)$ in a tubular channel for steady, fully developed laminar flow can be written in cylindrical coordinates as follows: $:^{[56,57]}$

$$
\begin{gathered}
v_{x}(r)=v_{\max }\left[1-\left(\frac{r}{R}\right)^{2}\right] \\
v_{\max }=2\left\langle v_{z}\right\rangle
\end{gathered}
$$

where $r$ is the cylindrical coordinate and $R$ is the channel radius. The representation of the flow for the considered tubular channel is illustrated in Fig. 1.6b. The velocity profile in a circular tube is parabolic, as for the parallel-plate channel, but the maximum velocity in this case is $2\langle v\rangle \cdot{ }^{[56]}$ Equation 25 was used by Schechter and Wissler in the modeling of a tubular photoreactor. ${ }^{[58]}$

The velocity profile $v_{z}(r)$ trough an annulus for steady-state, fully developed laminar flow can be written in cylindrical coordinates as follows: ${ }^{[57]}$

$$
\begin{gathered}
v_{z}(r)=v_{\max } \frac{1-\left(\frac{r}{r_{\text {ext }}}\right)^{2}+\frac{1-\kappa^{2}}{\ln \left(\frac{1}{\kappa}\right)} \ln \left(\frac{r}{r_{\text {ext }}}\right)}{\frac{1-\kappa^{4}}{1-\kappa^{2}}-\frac{1-\kappa^{2}}{\ln \left(\frac{1}{\kappa}\right)}} \\
v_{\max }=2\left\langle v_{z}\right\rangle
\end{gathered}
$$

where $r$ is the cylindrical coordinate, $r_{i n t}$ is the radius of the inner cylinder of the reactor, $r_{e x t}$ is the radius of the outer cylinder of the reactor, $\kappa$ is the ratio $r_{i n t} / r_{\text {ext }}$. The flow representation for the considered annulus is shown in Fig. 1.6c. Equation 27 was used in modeling annular photoreactors with small form variations. ${ }^{[28,45,51,55,59]}$

\subsection{Applications of continuous flow photoreactor modeling}

\subsubsection{Modeling for photoreactorscale-up}

Applications of the photoreactor scale-up methodology previously described are briefly presented in Table 1.2. Most of the applications are from the water and air treatment field, but the same scale-up methodology may be applied also in other areas, such as synthetic chemistry. As it can be observed, the methodology was used with various reactor geometries in homogeneous (liquid) and heterogeneous phase (gas-solid, liquid solid). The reported volumes of annular reactors range from $134 \mathrm{~cm}^{3}$ at laboratory scale ${ }^{[7]}$ and up to $6000 \mathrm{~cm}^{3}$ at large scale ${ }^{[6]}$. The flat-plate reactors were scaled from $26 \mathrm{~cm}^{3}$ at laboratory scale ${ }^{[49]}$ to up to $734 \mathrm{~cm}^{3}$ at large scale. ${ }^{[5]}$

The main purpose of this chapter was to demonstrate the use of modeling as a tool for the scale-up process, but it is also interesting to consider the use of modeling as a tool to investigate the behavior of photoreactors for reactor optimization, which we will do in the next section. 
Table 1.2. Examples of scale-up to large continuous photoreactors using the methodology reported by Alfano and Cassano ${ }^{[8]}$.

\begin{tabular}{lllll}
\hline Application & Medium & \multicolumn{2}{l}{ Reactor configuration } & \multirow{2}{*}{ Reference } \\
\cline { 2 - 4 } $\begin{array}{l}\text { Oxidation of dilute } \\
\text { aqueous HCOOH } \\
\text { solutions }\end{array}$ & Liquid & Flat-plate & Annular & \\
\hline $\begin{array}{l}\text { Degradation of } \\
\text { perchloroethylene }\end{array}$ & Gas-Solid & Flat plate & Multi-annular & ${ }^{[16]}$ \\
$\begin{array}{l}\text { Degradation of } 4- \\
\text { chlorophenol }\end{array}$ & $\begin{array}{l}\text { Liquid-Solid } \\
\text { (slurry) }\end{array}$ & Cylindrical & Flat-plate & ${ }^{[5]}$ \\
\hline $\begin{array}{l}\text { Degradation of } \\
\text { herbicides }\end{array}$ & $\begin{array}{l}\text { Liquid-Solid } \\
\text { (slurry) }\end{array}$ & Annular & Falling film & ${ }^{[7]}$ \\
\hline $\begin{array}{l}\text { Degradation of } \\
\text { formaldehyde }\end{array}$ & Gas-Solid & Flat-plate & Corrugated wall & ${ }^{[49]}$ \\
\hline $\begin{array}{l}\text { Inactivation of } \\
\text { Escherichia coli }\end{array}$ & $\begin{array}{l}\text { Liquid-Solid } \\
\text { (slurry) }\end{array}$ & Annular & Annular & ${ }^{[55]}$ \\
\hline $\begin{array}{l}\text { Degradation of } \\
\text { aqueous polyvinyl } \\
\text { alcohol }\end{array}$ & Liquid & Annular & Annular & ${ }^{[6]}$ \\
\hline
\end{tabular}

\subsubsection{Modeling for reactor optimization}

Similar to the previously discussed scale-up studies, modeling of the photoreactor behavior was mostly employed in the water and air treatment fields. In general, the classical photoreactor configurations are considered e.g., annular, flat-plate. However, recently also an increasing interest in the modeling of continuous-flow microreactors is observed.

One of the main objectives of the optimization investigations are the identification of mass transfer limitations (external and internal) and CFD is extensively used for predicting velocity fields, concentration gradients and the photoreaction rate.

Table 1.3 Example of applications of modeling for reactor optimization

\begin{tabular}{|c|c|c|c|}
\hline Application & Reactor & Investigation & Reference \\
\hline $\begin{array}{l}\text { Degradation of } \\
\text { trichloroethylene }\end{array}$ & Annular & $\begin{array}{l}\text { CFD modeling for predicting the performance of } \\
\text { various photocatalytic reactor concepts }\end{array}$ & \\
\hline $\begin{array}{l}\text { Degradation of an } \\
\text { organic pollutant }\end{array}$ & Annular & $\begin{array}{l}\text { Dependence of the degradation rate on dimensionless } \\
\text { parameters }\end{array}$ & [59] \\
\hline $\begin{array}{l}\text { Degradation of } \\
\text { dichloroacetic acid }\end{array}$ & Flat-plate & Mass transport limitations & {$[61,62]$} \\
\hline $\begin{array}{l}\text { Degradation of } \\
\text { ammonia and butiric } \\
\text { acid }\end{array}$ & Annular & Influence of mass transfer on kinetics & [63] \\
\hline $\begin{array}{l}\text { Degradation of } \\
\text { thrichloroethylene }\end{array}$ & Flat-plate & $\begin{array}{l}\text { CFD modeling to study the velocity field and } \\
\text { concentration gradient }\end{array}$ & [64] \\
\hline $\begin{array}{l}\text { Degradation of benzoic } \\
\text { acid }\end{array}$ & Annular & $\begin{array}{l}\text { CFD modeling to predict the degradation rate over } \\
\text { different operating regimes }\end{array}$ & [65] \\
\hline $\begin{array}{l}\text { Degradation of } \\
\text { shower water }\end{array}$ & Annular & $\begin{array}{l}\text { CFD modeling for relating the degradation rate with } \\
\text { operating regimes }\end{array}$ & [32] \\
\hline $\begin{array}{l}\text { Degradation of para- } \\
\text { chlorobenzoic acid }\end{array}$ & Annular & CFD modeling for predicting the degradation rate & [66] \\
\hline $\begin{array}{l}\text { Degradation of salicylic } \\
\text { acid }\end{array}$ & Microreactor & $\begin{array}{l}\text { CFD modeling for investigating the influence of reactor } \\
\text { geometry on photocatalytic efficiency }\end{array}$ & 7] \\
\hline $\begin{array}{l}\text { Non-catalyzed } \\
\text { reaction }\end{array}$ & Microreactor & $\begin{array}{l}\text { Diffusion limitations; guidelines for operating } \\
\text { conditions required for avoiding diffusion limitations }\end{array}$ & {$[13]$} \\
\hline $\begin{array}{l}\text { Degradation of } \\
\text { methylene blue }\end{array}$ & Flat-plate & CFD modeling for reactor optimization & [68] \\
\hline UV disinfection & Baffled cylindrical & CFD modeling for photoreactor optimization & [69] \\
\hline
\end{tabular}




\subsection{Conclusions}

The purpose of this chapter was to present the most reported and used modeling strategy to assist in the scale-up of photoreactors. We emphasized the interconnection between the principal steps of the approach, such as kinetic modeling, together with the radiation, material and momentum balances. In addition, we highlighted the most common assumptions to simplify the equations, which allows model development and obtaining results with reasonable computational effort.

The discussed examples from literature were chosen to illustrate the effect of the simplifications on the predicted results. This modeling methodology has been validated in various applications and for various reactor configurations and can therefore be considered as a reliable tool to guide the scale-up of continuous-flow photoreactors. Furthermore, these validated models also represent an important means in predicting photoreactor behavior.

\section{References}

[1] Hook, B. D. A.; Dohle, W.; Hirst, P. R.; Pickworth, M.; Berry, M. B.; Booker-Milburn, K. I. J. Org. Chem. 2005, 70 , $7558-7564$.

[2] Van Gerven, T.; Mul, G.; Moulijn, J.; Stankiewicz, A. Chem. Eng. Process. 2007, 46, 781-789.

[3] Su, Y.; Straathof, N. J. W.; Hessel, V.; Noël, T. Chem. Eur. J. 2014, 20, 10562-10589.

[4] Elgue, S.; Aillet, T.; Loubiere, K.; Conté, A.; Dechy-Cabaret, O.; Prat, L.; Horn, C. R.; Lobet, O.; Vallon, S. Chim.Oggi 2015, 33, 58-62.

[5] Satuf, M. L.; Brandi, R. J.; Cassano, A. E.; Alfano, O. M. Catal. Today 2007, 129, 110-117.

[6] Ghafoori, S.; Mehrvar, M.; Chan, P. K. Chem. Eng. J. 2014, 245, 133-142.

[7] Li Puma, G.; Toepfer, B.; Gora, A. Catal. Today 2007, 124, 124-132.

[8] Alfano, O. M.; Cassano, A. E. In Advances in Chemical Engineering; de Lasa, H. I., Serrano, B., Eds.; Elsevier, 2009; Vol. 36, pp 229-287.

[9] Alfano, O. M.; Romero, R. L.; Cassano, A. E. Chem. Eng. Sci. 1986, 41, 421-444.

[10] Cassano, A. E.; Martin, C. A.; Brandi, R. J.; Alfano, O. M. Ind. Eng. Chem. Res. 1995, 34, 2155-2201.

[11] Aillet, T.; Loubiere, K.; Dechy-Cabaret, O.; Prat, L. Chem. Eng. Process. Intensif. 2013, 64, $38-47$.

[12] Cassano, A. E.; Alfano, O. M. Catal. Today 2000, 58, 167-197.

[13] Aillet, T.; Loubière, K.; Prat, L. AIChE J. 2015, 61, 1284-1299.

[14] Serpone, N.; Salinaro, A. Pure Appl. Chem. 1999, 71, 303-320.

[15] Imoberdorf, G. E.; Irazoqui, H. A.; Cassano, A. E.; Alfano, O. M. Ind. Eng. Chem. Res. 2005, 44, 6075-6085.

[16] Imoberdorf, G. E.; Irazoqui, H. A.; Alfano, O. M.; Cassano, A. E. Chem. Eng. Sci. 2007, 62, 793-804.

[17] Ghafoori, S.; Mehrvar, M.; Chan, P. K. Ind. Eng. Chem. Res. 2012, 51, 14980-14993.

[18] Ghafoori, S.; Mehrvar, M.; Chan, P. K. Iran Polym J 2012, 21, 869-876.

[19] Marugán, J.; van Grieken, R.; Cassano, A. E.; Alfano, O. M. Appl. Catal., B. 2008, 85, 48-60.

[20] Pareek, V.; Chong, S.; Tadé, M.; Adesina, A. A. Asia-Pac.J.Chem.Eng. 2008, 3, 171-201.

[21] Li Puma, G.; Brucato, A. Catal. Today 2007, 122, 78-90.

[22] Pareek, V.; Brungs, M. P.; Adesina, A. A. Ind. Eng. Chem. Res. 2003, 42, 26-36.

[23] Romero, R. L.; Alfano, O. M.; Cassano, A. E. Ind. Eng. Chem. Res. 1997, 36, 3094-3109.

[24] Pareek, V. K.; Adesina, A. A. AIChE J. 2004, 50, 1273-1288.

[25] Mueses, M. A.; Machuca-Martinez, F.; Hernández-Ramirez, A.; Li Puma, G. Chem. Eng. J. 2015, 279, $442-451$.

[26] Zazueta, A. L. L.; Destaillats, H.; Li Puma, G. Chem. Eng. J. 2013, 217, 475-485.

[27] Aillet, T. Conception et mise en oeuvre de reacteurs photochimiques intensifies. Ph.D. Thesis, Institut National Polytechnique de Toulouse, 2015.

[28] Li Puma, G.; Khor, J.; Brucato, A. Environ. Sci. Technol. 2004, 38, 3737-3745.

[29] Brucato, A.; Cassano, A. E.; Grisafi, F.; Montante, G.; Rizzuti, L.; Vella, G. AIChE J. 2006, 52, 3882-3890.

[30] Grčić, I.; Li Puma, G. Environ. Sci. Technol. 2013, 47, 13702-13711.

[31] Sgalari, G.; Camera-Roda, G.; Santarelli, F. Int. Commun. Heat Mass 1998, 25, 651-660.

[32] Boyjoo, Y.; Ang, M.; Pareek, V. Chem. Eng. Sci. 2014, 111, 266-277.

[33] Spadoni, G.; Bandini, E.; Santarelli, F. Chem. Eng. Sci. 1978, 33, 517-524.

[34] Pasquali, M.; Santarelli, F.; Porter, J. F.; Yue, P.-L. AIChE J. 1996, 42, 532-537.

[35] Changrani, R.; Raupp, G. B. AIChE J. 1999, 45, 1085-1094.

[36] Imoberdorf, G. E.; Taghipour, F.; Keshmiri, M.; Mohseni, M. Chem. Eng. Sci. 2008, 63, 4228-4238. 
[37] Moreira, J.; Serrano, B.; Ortiz, A.; de Lasa, H. Ind. Eng. Chem. Res. 2010, 49, 10524-10534.

[38] Singh, M.; Salvado-Estivill, I.; Li Puma, G. AIChE J. 2007, 53, 678-686.

[39] Manassero, A.; Satuf, M. L.; Alfano, O. M. Environ. Sci. Pollut. Res. 2015, 22, 926-937.

[40] Pruvost, J.; Cornet, J. F.; Legrand, J. Chem. Eng. Sci. 2008, 63, 3679-3694.

[41] Motegh, M.; Cen, J.; Appel, P. W.; van Ommen, J. R.; Kreutzer, M. T. Chem. Eng. J. 2012, 207-208, 607-615.

[42] Mueses, M. A.; Machuca-Martinez, F.; Li Puma, G. Chem. Eng. J. 2013, 215-216, 937-947.

[43] Salvadó-Estivill, I.; Brucato, A.; Puma, G. L. Ind. Eng. Chem. Res. 2007, 46, 7489-7496.

[44] Visan, A.; Rafieian, D.; Ogieglo, W.; Lammertink, R. G. H. Appl. Catal., B 2014, 150-151, 93-100.

[45] Labas, M. D.; Zalazar, C. S.; Brandi, R. J.; Martín, C. A.; Cassano, A. E. Helv. Chim. Acta 2002, 85, 82-95.

[46] Queffeulou, A.; Geron, L.; Archambeau, C.; Le Gall, H.; Marquaire, P.-M.; Zahraa, O. Ind. Eng. Chem. Res. 2010, 49, 6890-6897.

[47] Brandi, R. J.; Rintoul, G.; Alfano, O. M.; Cassano, A. E. Catal. Today 2002, 76, 161-175.

[48] Salvadó-Estivill, I.; Hargreaves, D. M.; Puma, G. L. Environ. Sci. Technol. 2007, 41, 2028-2035.

[49] Passalía, C.; Alfano, O. M.; Brandi, R. J. J. Hazard. Mater. 2012, 211-212, 357-365.

[50] Camera-Roda, G.; Augugliaro, V.; Cardillo, A. .; Loddo, V.; Palmisano, L.; Parrino, F.; Santarelli, F. Catal. Today 2016, 259, 87-96.

[51] Marugán, J.; van Grieken, R.; Cassano, A. E.; Alfano, O. M. Catal. Today 2009, 144, 87-93.

[52] Gorges, R.; Meyer, S.; Kreisel, G. J. Photochem. Photobiol., A 2004, 167, 95-99.

[53] Charles, G.; Roques-Carmes, T.; Becheikh, N.; Falk, L.; Commenge, J.-M.; Corbel, S. J. Photochem. Photobiol., A 2011, 223, 202-211.

[54] Aillet, T.; Loubière, K.; Dechy-Cabaret, O.; Prat, L. Chem. Eng. Technol. 2016, 39, 115-122.

[55] Marugán, J.; van Grieken, R.; Pablos, C.; Satuf, M. L.; Cassano, A. E.; Alfano, O. M. Chem. Eng. J. 2013, 224, 3945.

[56] Deen, W. M. Analysis of transport phenomena, 2nd ed.; Oxford University Press USA, 2013.

[57] Bird, R. B.; Stewart, W. E.; Lightfoot, E. N. Transport phenomena, 2nd ed.; John Wiley \& Sons, Inc., 2002.

[58] Schechter, R. S.; Wissler, E. H. Appl. Sci. Res. 1960, 9, 334-344.

[59] Camera Roda, G.; Santarelli, F. Ind. Eng. Chem. Res. 2007, 46, 7637-7644.

[60] Taghipour, F.; Mohseni, M. AIChE J. 2005, 51, 3039-3047.

[61] Ballari, M. D. L. M.; Brandi, R.; Alfano, O.; Cassano, A. Chem. Eng. J. 2008, 136, 50-65.

[62] Ballari, M. D. L. M.; Brandi, R.; Alfano, O.; Cassano, A. Chem. Eng. J. 2008, 136, 242-255.

[63] Boulinguiez, B.; Bouzaza, A.; Merabet, S.; Wolbert, D. J. Photochem. Photobiol., A 2008, 200, $254-261$.

[64] Jarandehei, A.; De Visscher, A. AIChE J. 2009, 55, 312-320.

[65] Duran, J. E.; Mohseni, M.; Taghipour, F. AIChE J. 2011, 57, 1860-1872.

[66] Bagheri, M.; Mohseni, M. Chem. Eng. J. 2014, 256, 51-60.

[67] Corbel, S.; Becheikh, N.; Roques-Carmes, T.; Zahraa, O. Chem. Eng. Res. Des. 2014, 92, 657-662.

[68] Vaiano, V.; Sacco, O.; Pisano, D.; Sannino, D.; Ciambelli, P. Chem. Eng. Sci. 2015, 137, 152-160.

[69] Cao, H.; Deng, B.; Hong, J.; Xue, J.; Chang, F. Chem. Eng. Technol. 2016, 39, 108-114. 